\title{
A Comparative Study of Blindhide and Filterfirst Algorithm in Digital Images for Steganography Techniques
}

\author{
Purwantoro ${ }^{1}$, Garno $^{2}$, Munir $^{3}$, Ari Suntia ${ }^{4}$ \\ \{purwantoro.masbro@staff.unsika.ac.id ${ }^{1}$, garno@staff.unsika.ac.id ${ }^{2}$, munir@upi.edu ${ }^{3}$ \} \\ Universitas Singaperbangsa Karawang, Jl. H.S. Ronggowaluyo Telukjambe Karawang ${ }^{1,2}$, \\ Universitas Pendidikan Indonesia, J1 Dr. Setiabudi Isola Sukasari Bandung ${ }^{3,4}$
}

\begin{abstract}
The study was conducted in the field of hiding messages using the media image. This model is often referred to as steganography technique. Image media used for insertion are BMP (Bitmap Picture) format images, JPEG (Joint Photographic Expert Group) and PNG (Portable Network Graphics). The secret message form that is inserted is in the txt format. The application used for insertion is the Digital Invisible Ink Toolkit. Research parameters include image quality testing, and quantitative testing on each Blindhide algorithm and the Filterfirst algorithm. Image Quality test results using MSE, PSNR, showed significant differences, while the value of SC showed differences but not substantial. The results of testing and analysis evaluations that have been carried out on each image turned out to be an average value of the MSE value of the Blindhide algorithm 0.024 greater than the Filterfirst algorithm 0.023, PSNR Blindhide algorithm 31.91 smaller than the Filterfirst 32.03 algorithm, SC Blindhide algorithm 0.99986 smaller than the Filterfirst algorithm 0.99988. This shows that the insertion of a secret message into the image using the Filterfirst algorithm is safer and will not be suspected.
\end{abstract}

Keywords: Steganography, Embedding, Blindhide, Filterfirst, Digital Invisible Ink Toolkit

\section{Introduction}

Cryptography and steganography are techniques that are often used to secure and maintain the confidentiality of data. Cryptography focuses on protecting the contents of information so that it is secure and steganography focuses on transparently protecting data. The media used in steganography to hide messages and hidden media also varies, ranging from text, images (image), audio to video. But in its implementation, image media is one of the most widely used media in steganography, due to its many advantages.

Message storage media in steganography uses cover image media. The image that has been embedded in a message is called stegoimage and is measured for its quality. Stegoimage quality measurement results are the essential thing in knowing how well the steganography algorithm is used. The technique of measuring image quality can use two ways. The first way to measure image quality is done subjectively, namely the measurement of image quality is based on human perception. The second way, using an objective assessment, namely through quantitative measures, to produce standards that are at once the accuracy of the results of steganography. This Word document can be used as a template for papers to be published in 
EAI Core Proceedings. Follow the text for further instructions on text formatting, tables, figures, citations, and references.

Steganography as a message security technique must be resistant to attacks that try to find out the embedded message. The attack category is closed type when the original cover image is available before the word is inserted and the image is inserted or stegoimage. Both of these images can be analyzed by looking at the composition of colors, lighting, and pixels. Visual analysis affects the quality of steganographic results. Steganography techniques affect the quality of stegoimage. Many algorithms are used for research in steganography techniques. Each research technique provides its advantages.

Previous studies that carried out experiments with a steganographic algorithm gave various contributions to get the maximum value from a high similarity. So this research is intended to compare the method of blindhide and filterfirst steganography algorithms.

\section{Method}

The method described in the study includes two things, namely the message insertion technique using blindhide and filterfirst algorithms, test scenarios in filing technique.

\subsection{BlindHide}

BlindHide is the most straightforward technique for hiding information in an image, the algorithm can be seen in Figure 1. The method of hiding starts from the upper left corner of the bit in the picture. The technique works by walking to match the secret message bit characters into the corresponding image bit characters across the bits in the image then down through pixel by pixel. It runs along the significant bit change of the pixel color to match the message to be inserted[1].

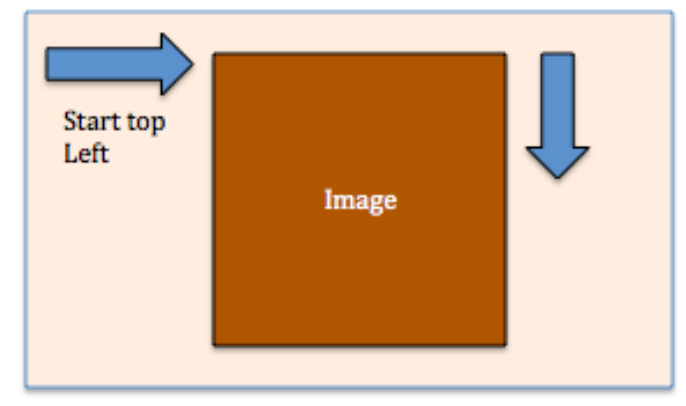

Fig. 1. Blindhide algorithm flow

The message insertion technique with the blindhide algorithm is as follows:

1) Choose a secret message

2) Change the secret message to a decimal value

3) Change the decimal value to an 8-bit binary number

4) Select a 24-bit image (BMP / JPEG / PNG)

5) Read the decimal value of the image matrix

6) Change back to 8 -bit binary numbers 
7) Image filtering

8) Read the image bit from the top left of the image

9) Insert message bits into the image bits with message bits starting from the left

10) Convert back the insertion value into a new image (stego)

\subsection{Filterfirst}

FilterFirst uses an edge-detecting filter, such as the Laplace formula, to find the areas of the image where there are pixels that are the least like their neighbours, this is ilustrated in Figure 2. It hides in the highest values of the filter first. Since we are only changing the $x$ least significant bits for steganography, we can use the $\mathrm{y}$ most significant bits for the filter. Here $\mathrm{x}$ and $y$ are integers where $1<=x<=7$ andy $=8-x$. We can guarantee that FilterFirst will be able to retrieve the information from the same pixels it hides in because the bits used in filtering are not changed by the hiding process. FilterFirst eliminates the need to provide any extra information, such as the original image, yet ensures the same pixels are used for hiding and retrieval[1,2].

\begin{tabular}{|r|r|r|r|}
\hline 1 & 2 & 3 & 4 \\
\hline 5 & 6 & 7 & 8 \\
\hline 9 & 10 & 11 & 12 \\
\hline 13 & 14 & 15 & 16 \\
\hline
\end{tabular}$\longrightarrow$\begin{tabular}{|r|r|r|r|}
\hline 5 & 6 & 7 & 8 \\
\hline 9 & 1 & 2 & 10 \\
\hline 11 & 3 & 4 & 12 \\
\hline 13 & 14 & 15 & 16 \\
\hline
\end{tabular}

Fig. 2. Filterfirst algorithm flow

The message insertion technique with the filterfirst algorithm is as follows:

1) Choose a secret message

2) Change the secret message to a decimal value

3) Change the decimal value to an 8-bit binary number

4) Select a 24-bit image (BMP / JPEG / PNG)

5) Read the decimal value of the image matrix

6) Change back to 8-bit binary numbers.

7) Image filtering

8) Looking for pixel image values from highest to lowest (from image filters)

9) Insert message bits into selected image bits with message bits starting from the far left

10) Convert back the insertion value into a new image (stego)

\subsection{Test Scenario}

Test methods performed can be seen in accordance with the following Figure 3 . 


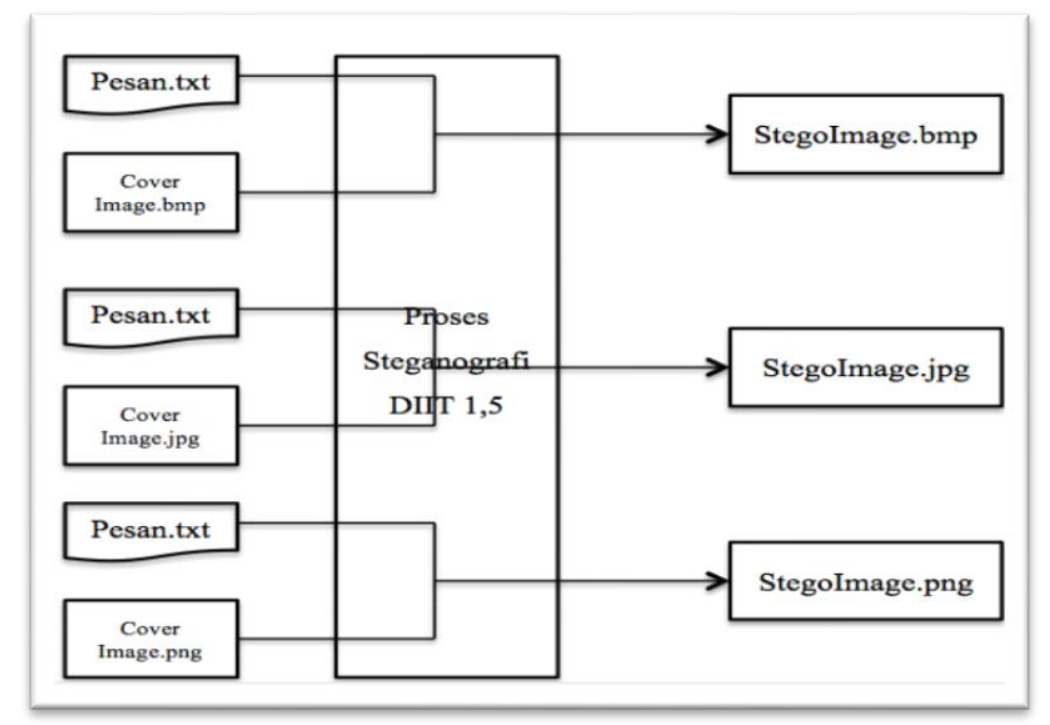

Fig. 3. Test methods.

Figure 3 above provides information on the insertion process flow. An image file format used by BMP, JPEG, PNG has inserted a secret message with the Txt format. Message insertion is done using the Digital Invisible Ink Toolkit 1.5 application. The algorithm used is Blindhide and Filter first. Test results compared with the original image. The algorithm that produces the stego image most similar to the original image is the best.

\section{Experimental Results}

Cover image in this study is the image with $B M P, J P E G$, and $P N G$ formats. The test sample in this study used message.txt and 30 images in each format. Table 1 below presents a cover image as a population and research sample.

\subsection{Cover image}

Table 1. Cover image population.

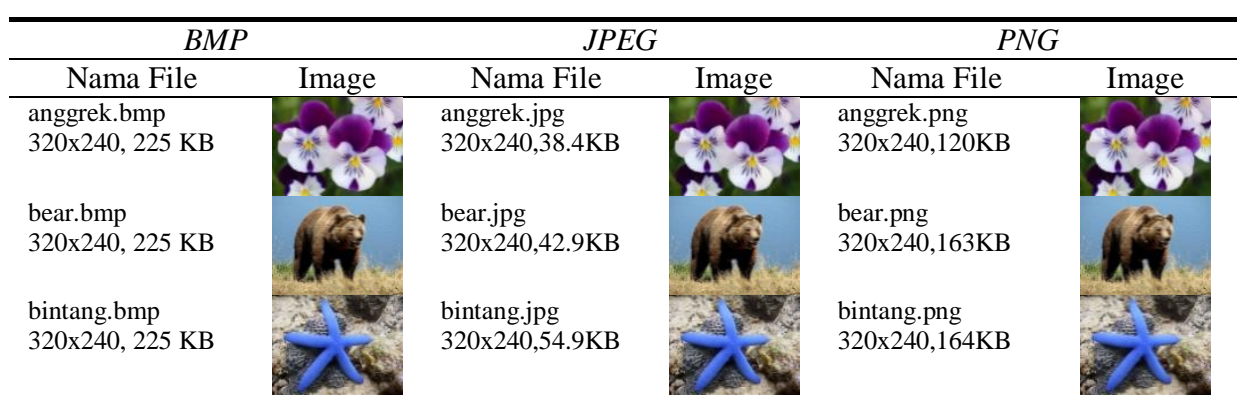




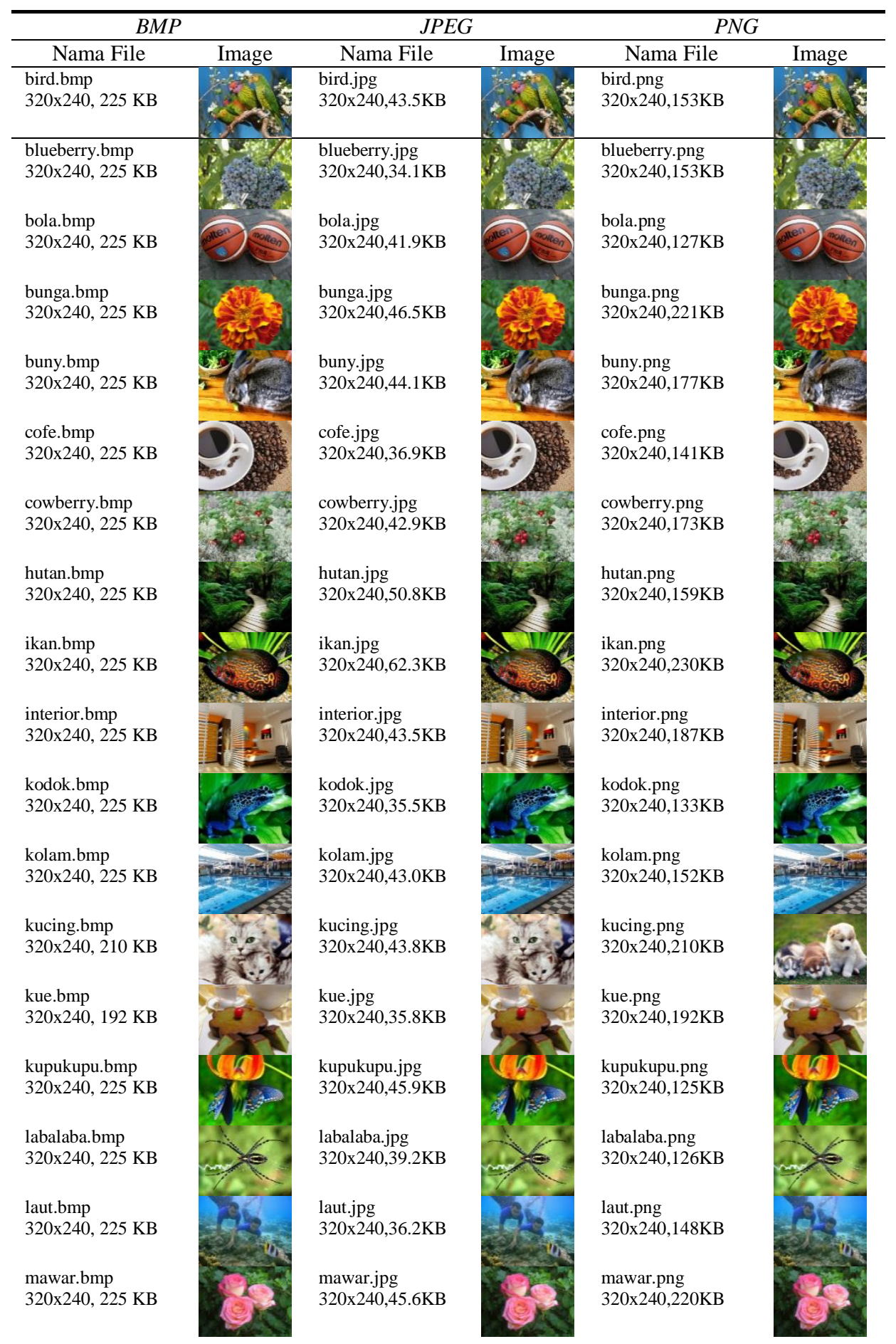




\begin{tabular}{|c|c|c|c|c|c|}
\hline \multicolumn{2}{|c|}{$B M P$} & \multicolumn{2}{|c|}{$J P E G$} & \multicolumn{2}{|c|}{$P N G$} \\
\hline Nama File & Image & Nama File & Image & Nama File & Image \\
\hline $\begin{array}{l}\text { mobil.bmp } \\
320 \times 240,225 \mathrm{~KB}\end{array}$ & & $\begin{array}{l}\text { mobil.jpg } \\
320 \times 240,44.8 \mathrm{~KB}\end{array}$ & & $\begin{array}{l}\text { mobil.png } \\
320 \times 240,136 \mathrm{~KB}\end{array}$ & \\
\hline $\begin{array}{l}\text { pantai.bmp } \\
320 \times 240,225 \mathrm{~KB}\end{array}$ & & $\begin{array}{l}\text { pantai.jpg } \\
320 \times 240,43.9 \mathrm{~KB}\end{array}$ & & $\begin{array}{l}\text { pantai.png } \\
320 \times 240,136 \mathrm{~KB}\end{array}$ & \\
\hline $\begin{array}{l}\text { pemandangan.bmp } \\
320 \times 240,225 \mathrm{~KB}\end{array}$ & & $\begin{array}{l}\text { pemandangan.jpg } \\
320 \times 240,38.4 \mathrm{~KB}\end{array}$ & & $\begin{array}{l}\text { pemandangan.png } \\
320 \times 240,161 \mathrm{~KB}\end{array}$ & \\
\hline $\begin{array}{l}\text { puppy.bmp } \\
320 \times 240,225 \mathrm{~KB}\end{array}$ & & $\begin{array}{l}\text { puppy.jpg } \\
320 \times 240,40.1 \mathrm{~KB}\end{array}$ & & $\begin{array}{l}\text { puppy.png } \\
320 \times 240,210 \mathrm{~KB}\end{array}$ & \\
\hline $\begin{array}{l}\text { strawberry.bmp } \\
320 \times 240,225 \mathrm{~KB}\end{array}$ & & $\begin{array}{l}\text { strawberry.jpg } \\
320 \times 240,61 \mathrm{~KB}\end{array}$ & & $\begin{array}{l}\text { strawberry.png } \\
320 \times 240,164 \mathrm{~KB}\end{array}$ & \\
\hline $\begin{array}{l}\text { sungai.bmp } \\
320 \times 240,225 \mathrm{~KB}\end{array}$ & & $\begin{array}{l}\text { sungai.jpg } \\
320 \times 240,39.6 \mathrm{~KB}\end{array}$ & & $\begin{array}{l}\text { sungai.png } \\
320 \times 240,176 \mathrm{~KB}\end{array}$ & \\
\hline $\begin{array}{l}\text { teddy.bmp } \\
320 \times 240,225 \mathrm{~KB}\end{array}$ & & $\begin{array}{l}\text { teddy.jpg } \\
320 \times 240,34.5 \mathrm{~KB}\end{array}$ & & $\begin{array}{l}\text { teddy.png } \\
320 \times 240,93 \cdot 6 \mathrm{~KB}\end{array}$ & \\
\hline $\begin{array}{l}\text { tomat.bmp } \\
320 \times 240,225 \mathrm{~KB}\end{array}$ & & $\begin{array}{l}\text { tomat.jpg } \\
320 \times 240,35.9 \mathrm{~KB}\end{array}$ & & $\begin{array}{l}\text { tomat.png } \\
320 \times 240,193 \mathrm{~KB}\end{array}$ & \\
\hline $\begin{array}{l}\text { wortel.bmp } \\
320 \times 240,225 \mathrm{~KB}\end{array}$ & & $\begin{array}{l}\text { wortel.jpg } \\
320 \times 240,53.6 \mathrm{~KB}\end{array}$ & & $\begin{array}{l}\text { wortel.png } \\
320 \times 240,180 \mathrm{~KB}\end{array}$ & \\
\hline
\end{tabular}

\subsection{Inserting message.txt into image.bmp.}

Embedding the message.txt to the image will resulting in Table 2.

Table 2. Embedding message to image.bmp.

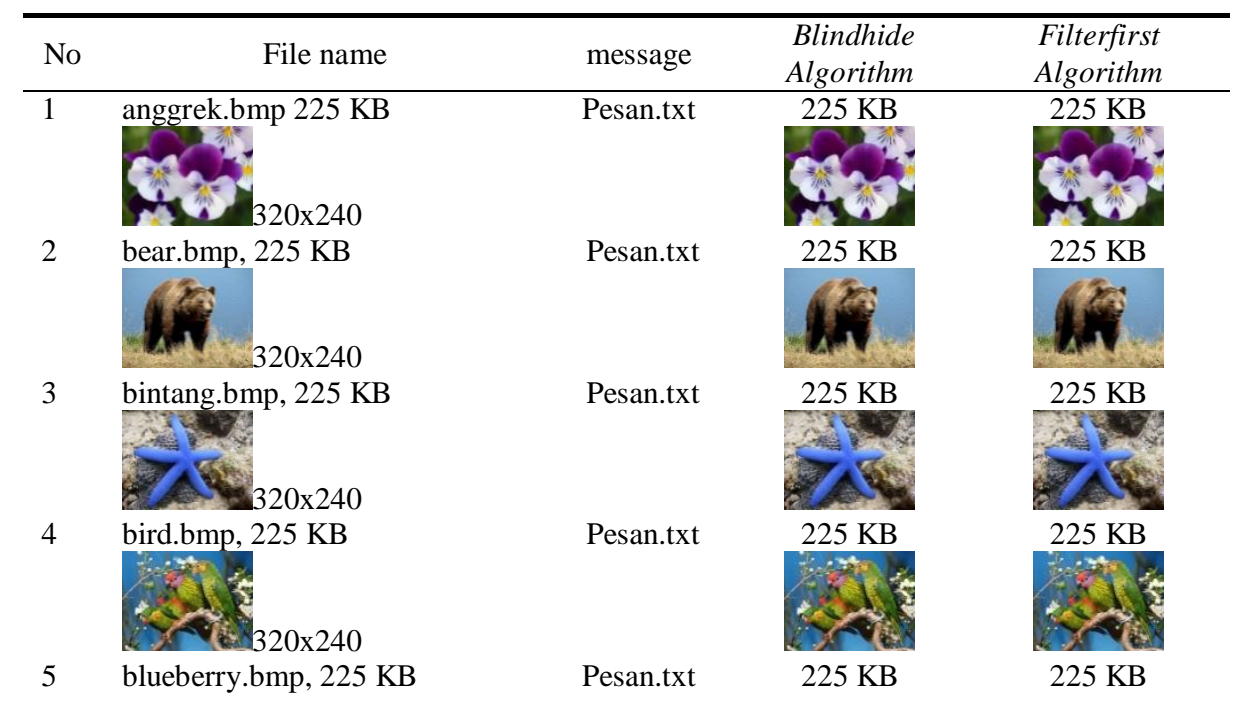




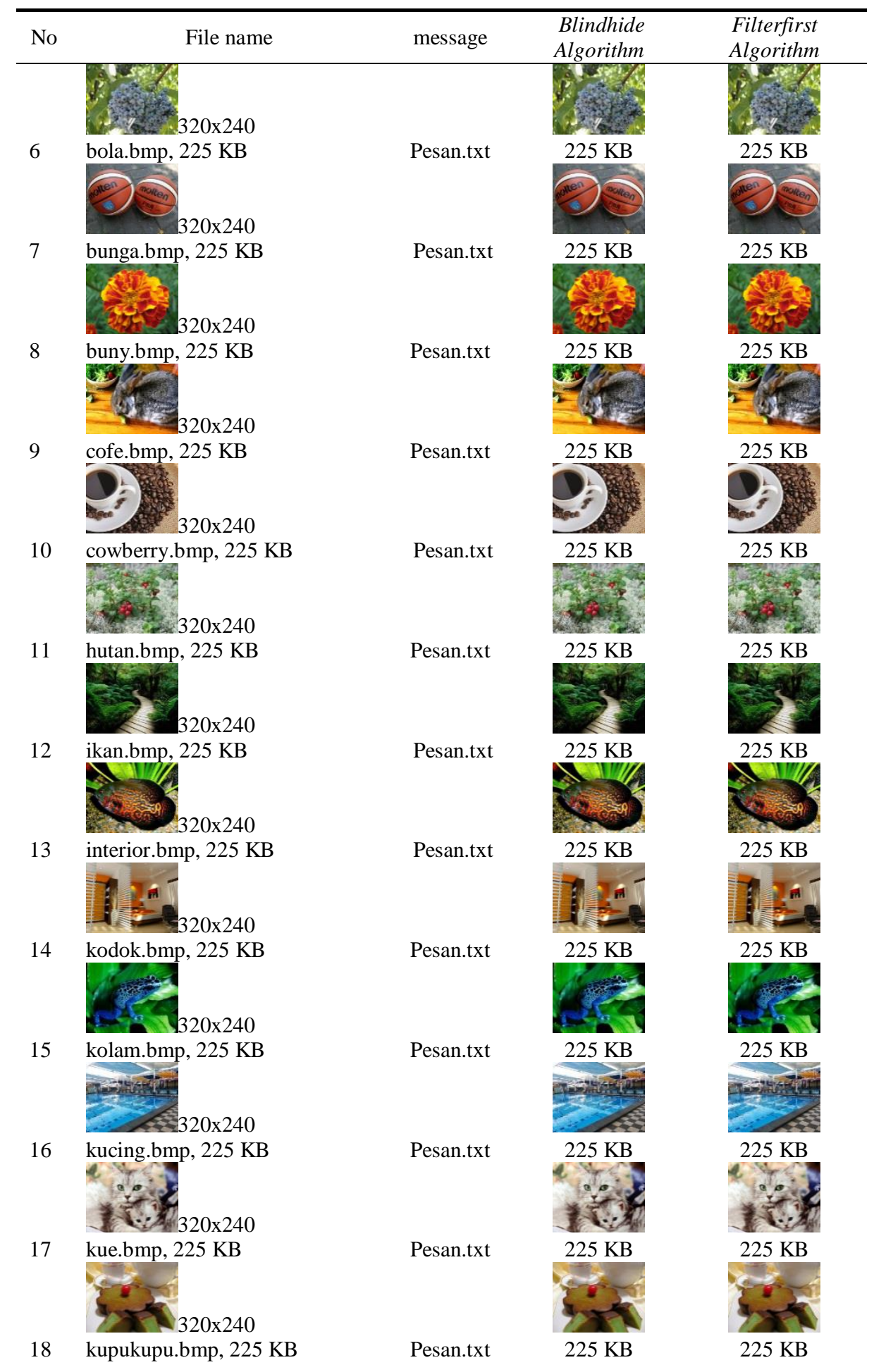




\begin{tabular}{|c|c|c|c|c|}
\hline No & File name & message & $\begin{array}{l}\text { Blindhide } \\
\text { Algorithm }\end{array}$ & $\begin{array}{l}\text { Filterfirst } \\
\text { Algorithm }\end{array}$ \\
\hline & & & & \\
\hline 19 & labalaba.bmp, $225 \mathrm{~KB}$ & Pesan.txt & $225 \mathrm{~KB}$ & $225 \mathrm{~KB}$ \\
\hline 20 & laut.bmp, $225 \mathrm{~KB}$ & Pesan.txt & $225 \mathrm{~KB}$ & $225 \mathrm{~KB}$ \\
\hline 21 & mawar.bmp, $225 \mathrm{~KB}$ & Pesan.txt & $225 \mathrm{~KB}$ & $225 \mathrm{~KB}$ \\
\hline 22 & mobil.bmp, $225 \mathrm{~KB}$ & Pesan.txt & $225 \mathrm{~KB}$ & \\
\hline 23 & pantai.bmp, $225 \mathrm{~KB}$ & Pesan.txt & $225 \mathrm{~KB}$ & $225 \mathrm{~KB}$ \\
\hline 24 & pemandangan.bmp, $225 \mathrm{~KB}$ & Pesan.txt & $225 \mathrm{~KB}$ & $225 \mathrm{~KB}$ \\
\hline 25 & puppy.bmp, $225 \mathrm{~KB}$ & Pesan.txt & $225 \mathrm{~KB}$ & \\
\hline & vences $320 x 240$ & & & \\
\hline 26 & y.bmp, $225 \mathrm{~KB}$ & Pesan.txt & & \\
\hline 27 & sungai.bmp, $225 \mathrm{~KB}$ & Pesan.txt & $225 \mathrm{~KB}$ & $225 \mathrm{~KB}$ \\
\hline 28 & teddy.bmp, $225 \mathrm{~KB}$ & Pesan.txt & $225 \mathrm{~KB}$ & $225 \mathrm{~KB}$ \\
\hline 29 & tomat.bmp, $225 \mathrm{~KB}$ & Pesan.txt & $225 \mathrm{~KB}$ & $225 \mathrm{~KB}$ \\
\hline 30 & wortel.bmp, $225 \mathrm{~KB}$ & Pesan.txt & $225 \mathrm{~KB}$ & $225 \mathrm{~KB}$ \\
\hline
\end{tabular}




\subsection{Inserting message.txt into image.jpg}

Embedding the message.txt to the image will resulting in Table 3.

Table 3. Embedding Message to Image.jpg.

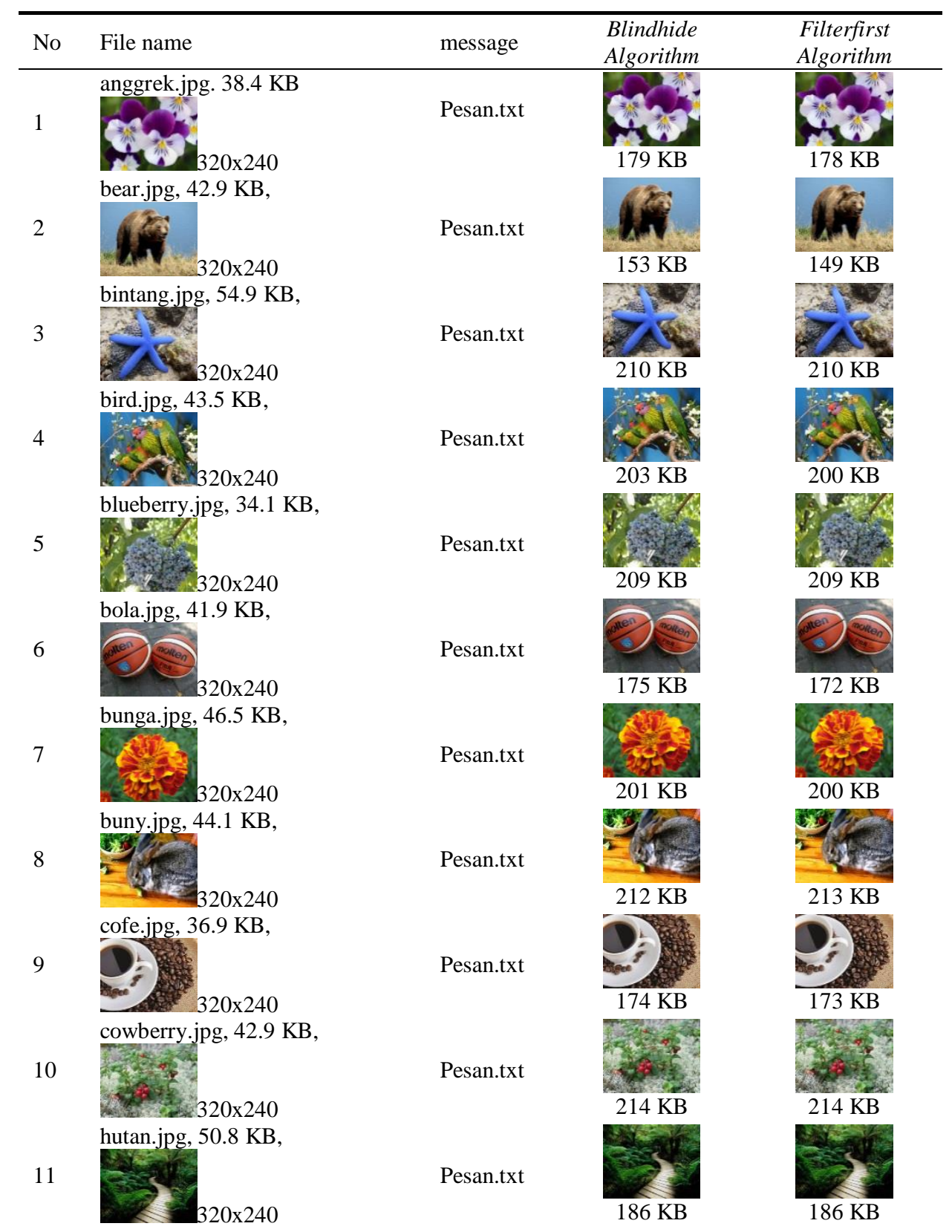




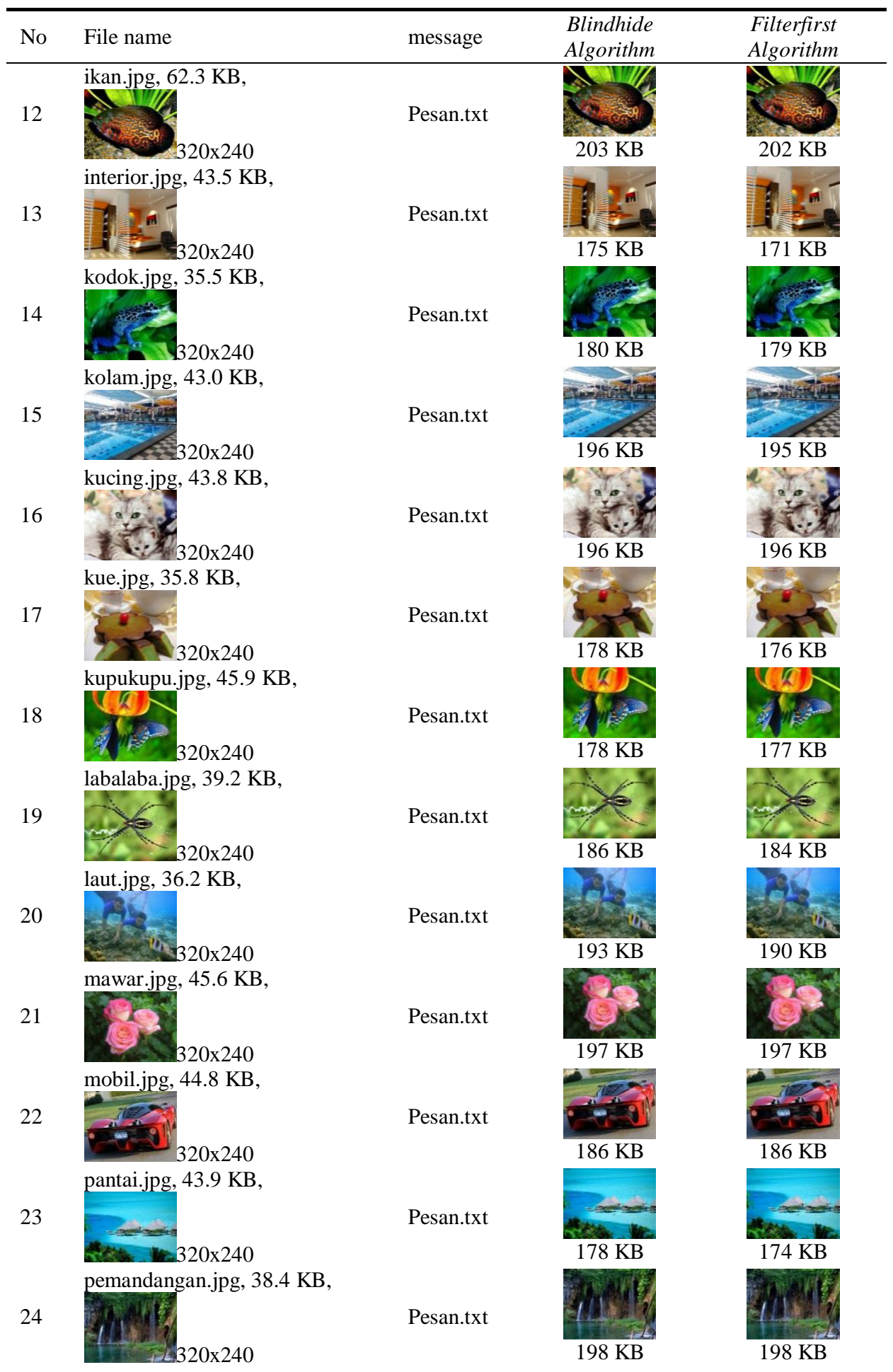




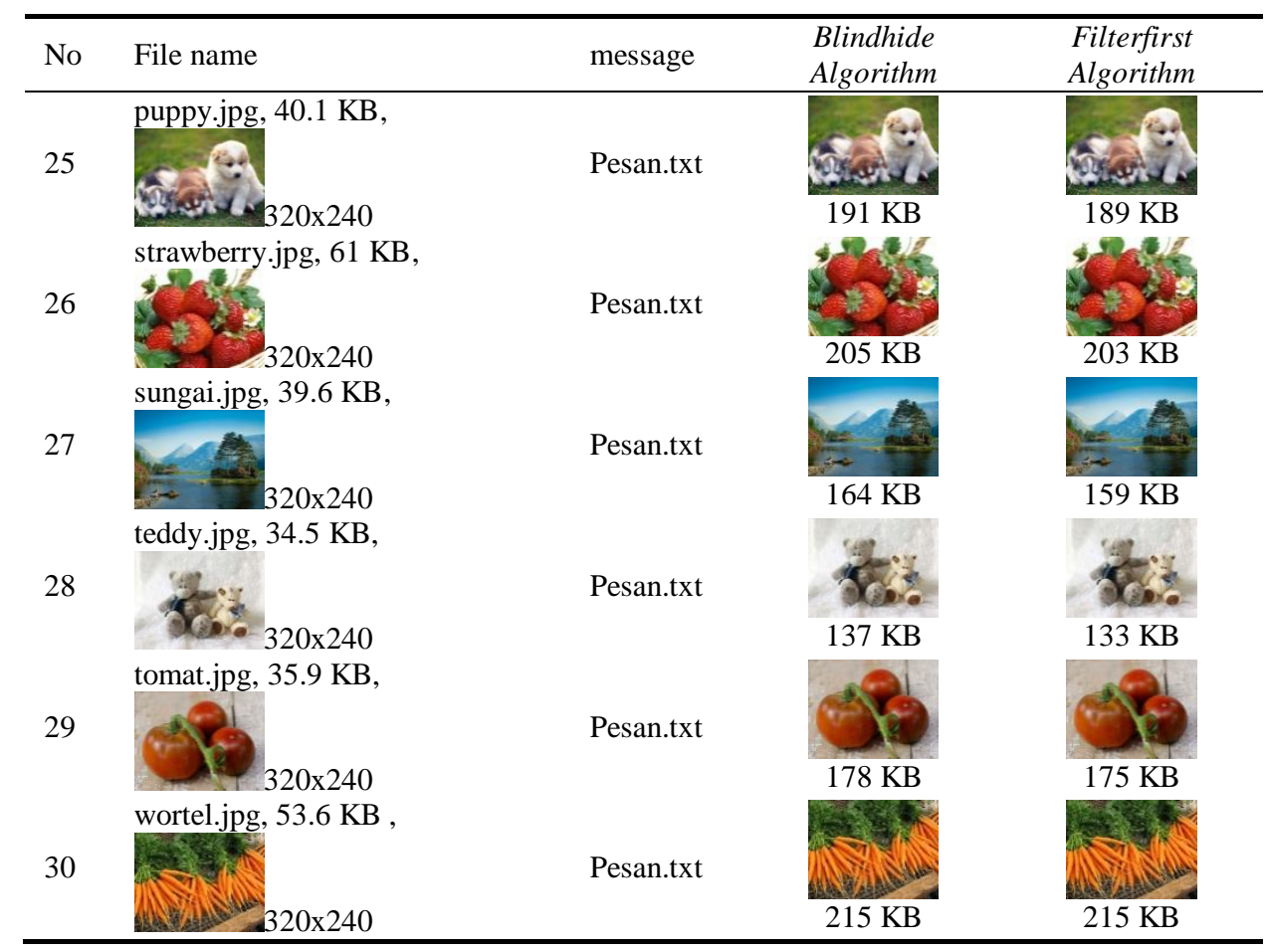

\subsection{Inserting message.txt into image.png}

Embedding the message.txt to the image will resulting in Table 4.

Table 4. Embedding Message to Image.png.

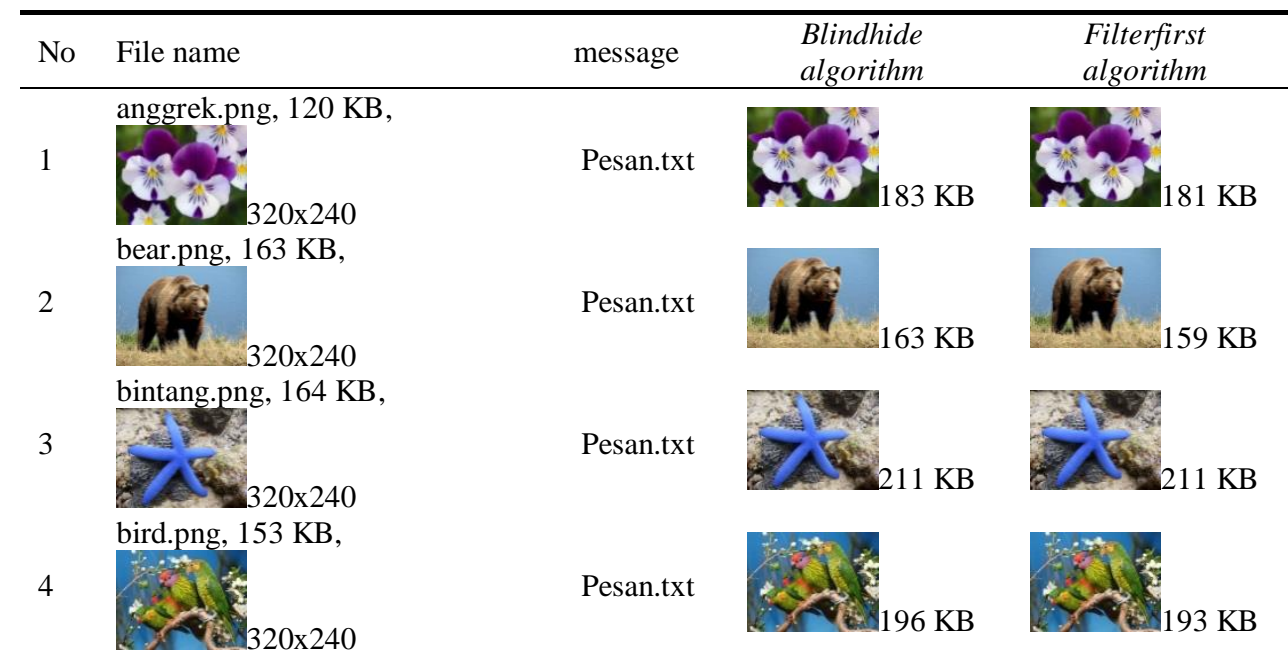




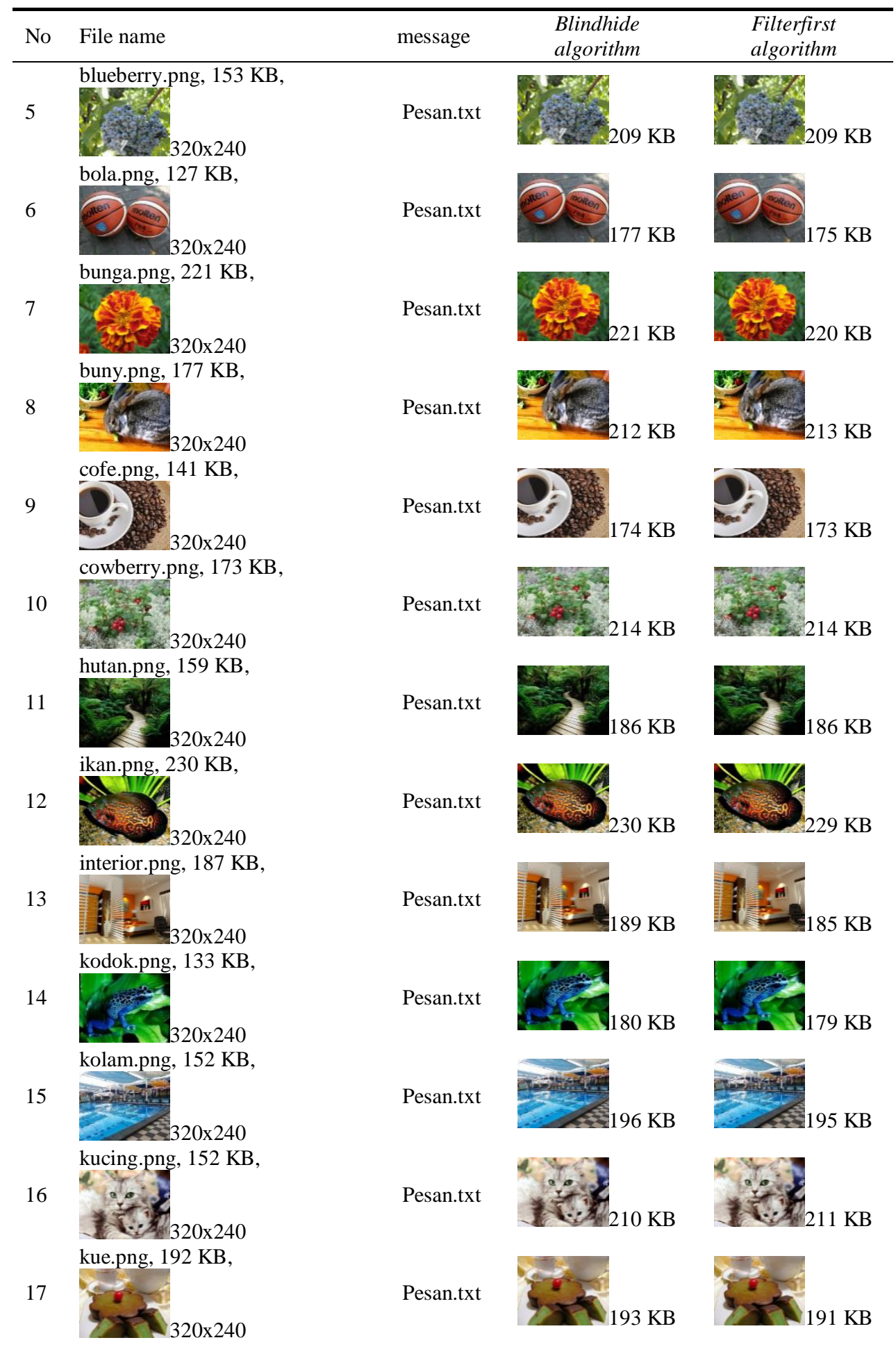




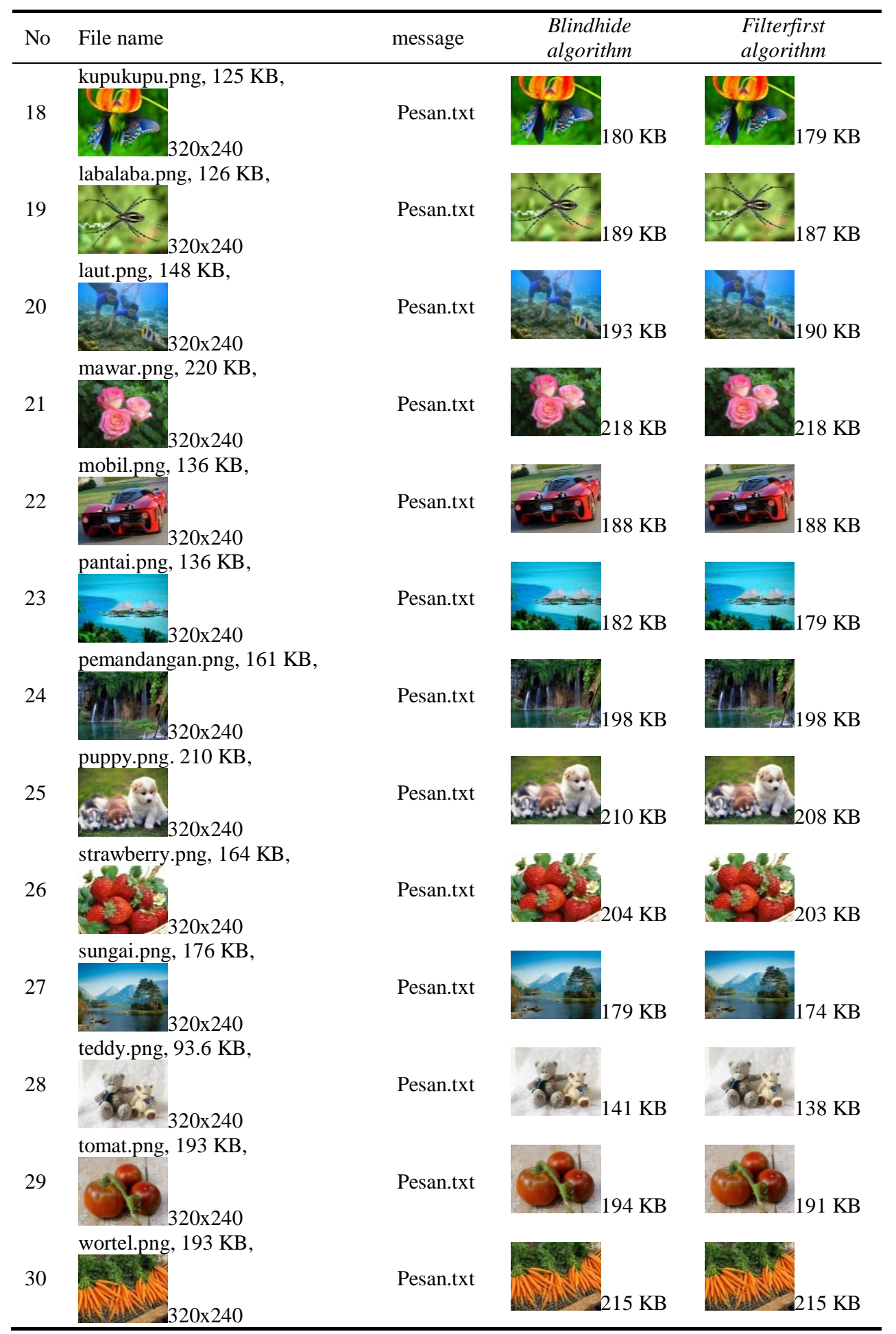




\subsection{MSE image value bmp jpg png}

Table 5 below is describing the MSE image value.

Table 5. MSE test results.

\begin{tabular}{|c|c|c|c|c|c|c|c|c|c|c|}
\hline \multirow{2}{*}{ No } & \multirow{2}{*}{ filename } & \multicolumn{3}{|c|}{ bmp } & \multicolumn{3}{|c|}{ jpg } & \multicolumn{3}{|c|}{ png } \\
\hline & & $c i$ & $b h$ & $f f$ & $c i$ & $b h$ & $f f$ & $c i$ & $b h$ & $f f$ \\
\hline 1 & anggrek & 0 & 0.024 & 0.023 & 0 & 0.025 & 0.024 & 0 & 0.024 & 0.023 \\
\hline 2 & bear & 0 & 0.026 & 0.024 & 0 & 0.025 & 0.024 & 0 & 0.026 & 0.024 \\
\hline 3 & bintang & 0 & 0.025 & 0.024 & 0 & 0.024 & 0.023 & 0 & 0.025 & 0.024 \\
\hline 4 & bird & 0 & 0.024 & 0.024 & 0 & 0.024 & 0.024 & 0 & 0.024 & 0.024 \\
\hline 5 & blueberry & 0 & 0.024 & 0.024 & 0 & 0.024 & 0.024 & 0 & 0.024 & 0.024 \\
\hline 6 & bola & 0 & 0.025 & 0.024 & 0 & 0.024 & 0.024 & 0 & 0.025 & 0.024 \\
\hline 7 & bunga & 0 & 0.024 & 0.025 & 0 & 0.024 & 0.026 & 0 & 0.024 & 0.025 \\
\hline 8 & buny & 0 & 0.024 & 0.024 & 0 & 0.024 & 0.024 & 0 & 0.024 & 0.024 \\
\hline 9 & cofe & 0 & 0.024 & 0.023 & 0 & 0.024 & 0.023 & 0 & 0.024 & 0.023 \\
\hline 10 & cowberry & 0 & 0.025 & 0.024 & 0 & 0.024 & 0.023 & 0 & 0.024 & 0.024 \\
\hline 11 & hutan & 0 & 0.023 & 0.022 & 0 & 0.024 & 0.023 & 0 & 0.023 & 0.022 \\
\hline 12 & ikan & 0 & 0.024 & 0.024 & 0 & 0.024 & 0.024 & 0 & 0.024 & 0.024 \\
\hline 13 & interior & 0 & 0.025 & 0.024 & 0 & 0.024 & 0.024 & 0 & 0.025 & 0.024 \\
\hline 14 & kodok & 0 & 0.021 & 0.02 & 0 & 0.021 & 0.02 & 0 & 0.021 & 0.02 \\
\hline 15 & kolam & 0 & 0.024 & 0.023 & 0 & 0.024 & 0.023 & 0 & 0.024 & 0.023 \\
\hline 16 & kucing & 0 & 0.024 & 0.024 & 0 & 0.024 & 0.024 & 0 & 0.024 & 0.024 \\
\hline 17 & kue & 0 & 0.024 & 0.024 & 0 & 0.024 & 0.024 & 0 & 0.024 & 0.024 \\
\hline 18 & kupukupu & 0 & 0.026 & 0.024 & 0 & 0.026 & 0.024 & 0 & 0.026 & 0.024 \\
\hline 19 & labalaba & 0 & 0.024 & 0.022 & 0 & 0.024 & 0.022 & 0 & 0.024 & 0.022 \\
\hline 20 & laut & 0 & 0.024 & 0.024 & 0 & 0.025 & 0.025 & 0 & 0.024 & 0.024 \\
\hline 21 & mawar & 0 & 0.024 & 0.026 & 0 & 0.024 & 0.025 & 0 & 0.024 & 0.026 \\
\hline 22 & mobil & 0 & 0.025 & 0.024 & 0 & 0.024 & 0.023 & 0 & 0.025 & 0.024 \\
\hline 23 & pantai & 0 & 0.024 & 0.022 & 0 & 0.023 & 0.02 & 0 & 0.024 & 0.022 \\
\hline 24 & pemandangan & 0 & 0.023 & 0.023 & 0 & 0.023 & 0.022 & 0 & 0.023 & 0.023 \\
\hline 25 & puppy & 0 & 0.025 & 0.023 & 0 & 0.025 & 0.024 & 0 & 0.025 & 0.023 \\
\hline 26 & strawberry & 0 & 0.033 & 0.028 & 0 & 0.033 & 0.027 & 0 & 0.033 & 0.028 \\
\hline 27 & sungai & 0 & 0.025 & 0.023 & 0 & 0.021 & 0.023 & 0 & 0.025 & 0.023 \\
\hline 28 & teddy & 0 & 0.024 & 0.024 & 0 & 0.024 & 0.023 & 0 & 0.024 & 0.024 \\
\hline 29 & tomat & 0 & 0.024 & 0.024 & 0 & 0.023 & 0.023 & 0 & 0.024 & 0.024 \\
\hline 30 & wortel & 0 & 0.025 & 0.023 & 0 & 0.025 & 0.022 & 0 & 0.025 & 0.023 \\
\hline
\end{tabular}

PSNR value of BMP original images with an average of $99 \mathrm{db}$. The average PSNR value of the stego image from the Blindhide algorithm is $31,939 \mathrm{db}$ and the Filterfirst algorithm is 32,049 db. PSNR value of Jpg original image with an average of $99 \mathrm{db}$. The average PSNR value of the stego image from the Blindhide algorithm is $31.88 \mathrm{db}$ and the Filterfirst algorithm is $32.00 \mathrm{db}$. PSNR value of Png original image with an average of $99 \mathrm{db}$. The average PSNR value of the stego image from the Blindhide algorithm is 31,906 $\mathrm{db}$ and the Filterfirst algorithm is $32,048 \mathrm{db}$.

\subsection{SC image value bmp jpg png}


Table 6 below is describing the SC image value.

Table 6. SC test results.

\begin{tabular}{|c|c|c|c|c|c|c|c|c|c|c|}
\hline \multirow{2}{*}{$\mathrm{No}$} & \multirow{2}{*}{ filename } & \multicolumn{3}{|c|}{$b m p$} & \multicolumn{3}{|c|}{ jpg } & \multicolumn{3}{|c|}{$p n g$} \\
\hline & & $c i$ & $b h$ & $f f$ & $c i$ & $b h$ & $f f$ & $c i$ & $b h$ & $f f$ \\
\hline 1 & anggrek & 1 & 0.9999 & 0.9999 & 1 & 0.9999 & 0.9999 & 1 & 0.9999 & 0.9999 \\
\hline 2 & bear & 1 & 0.9998 & 0.9999 & 1 & 0.9999 & 0.9999 & 1 & 0.9998 & 0.9999 \\
\hline 3 & bintang & 1 & 0.9999 & 0.9999 & 1 & 0.9999 & 0.9999 & 1 & 0.9999 & 0.9999 \\
\hline 4 & bird & 1 & 0.9998 & 0.9999 & 1 & 0.9999 & 0.9999 & 1 & 0.9998 & 0.9999 \\
\hline 5 & blueberry & 1 & 0.9998 & 0.9999 & 1 & 0.9999 & 0.9999 & 1 & 0.9999 & 0.9999 \\
\hline 6 & bola & 1 & 0.9999 & 0.9999 & 1 & 0.9999 & 0.9999 & 1 & 0.9999 & 0.9999 \\
\hline 7 & bunga & 1 & 0.9999 & 0.9999 & 1 & 0.9999 & 0.9998 & 1 & 0.9999 & 0.9999 \\
\hline 8 & buny & 1 & 0.9999 & 0.9999 & 1 & 0.9999 & 0.9999 & 1 & 0.9999 & 0.9999 \\
\hline 9 & cofe & 1 & 0.9999 & 0.9999 & 1 & 0.9999 & 0.9999 & 1 & 0.9999 & 0.9999 \\
\hline 10 & cowberry & 1 & 0.9999 & 0.9999 & 1 & 0.9999 & 0.9999 & 1 & 0.9999 & 0.9999 \\
\hline 11 & hutan & 1 & 0.9998 & 0.9998 & 1 & 0.9998 & 0.9998 & 1 & 0.9998 & 0.9998 \\
\hline 12 & ikan & 1 & 0.9999 & 0.9999 & 1 & 0.9999 & 0.9998 & 1 & 0.9999 & 0.9999 \\
\hline 13 & interior & 1 & 0.9999 & 0.9999 & 1 & 0.9999 & 0.9999 & 1 & 0.9999 & 0.9999 \\
\hline 14 & kodok & 1 & 1 & 1 & 1 & 1 & 1 & 1 & 1 & 1 \\
\hline 15 & kolam & 1 & 0.9999 & 0.9999 & 1 & 0.9998 & 0.9999 & 1 & 0.9999 & 0.9999 \\
\hline 16 & kucing & 1 & 0.9999 & 0.9999 & 1 & 0.9999 & 0.9999 & 1 & 0.9999 & 0.9999 \\
\hline 17 & kue & 1 & 0.9999 & 0.9999 & 1 & 0.9999 & 0.9999 & 1 & 0.9999 & 0.9999 \\
\hline 18 & kupukupu & 1 & 0.9998 & 0.9998 & 1 & 0.9998 & 0.9998 & 1 & 0.9998 & 0.9998 \\
\hline 19 & labalaba & 1 & 0.9999 & 0.9999 & 1 & 0.9999 & 0.9999 & 1 & 0.9999 & 0.9999 \\
\hline 20 & laut & 1 & 0.9998 & 0.9998 & 1 & 0.9998 & 0.9998 & 1 & 0.9999 & 0.9998 \\
\hline 21 & mawar & 1 & 0.9999 & 0.9998 & 1 & 0.9999 & 0.9998 & 1 & 0.9999 & 0.9998 \\
\hline 22 & mobil & 1 & 0.9999 & 0.9999 & 1 & 0.9999 & 0.9999 & 1 & 0.9999 & 0.9999 \\
\hline 23 & pantai & 1 & 0.9997 & 0.9999 & 1 & 0.9998 & 1 & 1 & 0.9997 & 0.9999 \\
\hline 24 & pemandangan & 1 & 0.9998 & 0.9998 & 1 & 0.9998 & 0.9998 & 1 & 0.9998 & 0.9998 \\
\hline 25 & puppy & 1 & 0.9999 & 0.9999 & 1 & 0.9999 & 0.9999 & 1 & 0.9999 & 0.9999 \\
\hline 26 & strawberry & 1 & 0.9997 & 0.9998 & 1 & 0.9997 & 0.9998 & 1 & 0.9997 & 0.9998 \\
\hline 27 & sungai & 1 & 0.9998 & 0.9999 & 1 & 1 & 0.9999 & 1 & 0.9998 & 0.9999 \\
\hline 28 & teddy & 1 & 0.9999 & 0.9999 & 1 & 0.9999 & 0.9999 & 1 & 0.9999 & 0.9999 \\
\hline 29 & tomat & 1 & 0.9999 & 0.9999 & 1 & 0.9999 & 0.9999 & 1 & 0.9999 & 0.9999 \\
\hline 30 & wortel & 1 & 0.9999 & 0.9999 & 1 & 0.9999 & 0.9999 & 1 & 0.9999 & 0.9999 \\
\hline
\end{tabular}

The SC value of original BMP images with an average of 1 . The average SC value of the stego image of the Blindhide algorithm is 0.99986 and the Filterfirst algorithm is 0.99988 . SC value of Jpeg original image with an average of 1 . The average SC value of the stego image of the Blindhide algorithm is 0.9998 and the Filterfirst algorithm is 0.9998. SC value of Png original image with an average of 1 . The average SC value of the stego image of the Blindhide algorithm is 0.99987 and the Filterfirst algorithm is 0.99988.

\section{Conclution}

The results of testing the original image with a stego image showed a slight change in the frequency of each color. The results of testing and analysis evaluations that have been carried out on each image turned out to be an average value of the MSE value of the Blindhide algorithm 0.024 greater than the Filterfirst algorithm 0.023, PSNR Blindhide algorithm 31.91 
smaller than the Filterfirst 32.03 algorithm, SC Blindhide algorithm 0.99986 smaller than the Filterfirst algorithm 0.99988 . This shows that the insertion of a secret message into the image using the Filterfirst algorithm is safer and will not be suspected.

\section{References}

[1] Rana, M.: Parameter Evaluation and Comparison of Algorithms Used In Steganography. International Journal of Engineering Science and Computing . pp. 8137 (2016)

[2] Hempstalk, K.: Hiding Behind Corners: Using Edges in Images for Better Steganography. https://www.researchgate.net/publication/241605558 (2014)

[3] Suryani, H.: Metode Riset Kuantitatif. Penadamedia Group. Jakarta, Indonesia (2015)

[4] Andono, T. M.: Pengolahan Citra Digital. Andi. Yogyakarta, Indonesia (2017)

[5] Zam, E.: Melacak, Membajak \& Membobol Data Rahasia. Mediakita. South Jakarta, Indonesia (2013)

[6] Sahyar, M. M.: Algoritma dan Pemprograman Menggunakan Matlab (Matrix Laboratory). Kencana. Jakarta, Indonesia (2016)

[7] Indriyono, B. V.: Implementasi Sistem Keamanan File dengan Metode Steganografi EOF dan Enkripsi Caesar Cipher. jurnal SISFO. pp. 4-5 (2016)

[8] Hidayatullah, P.: Pengolahan Citra Digital - Teori dan Aplikasi Nyata. Informatika. Bandung, Indonesia (2017)

[9] Bahrudiin, A. S.: Metode Penelitian Kuantitatif Aplikasi dalam Pendidikan. Deepublish. Yogyakarta (2014)

[10] Munir, R.: Kriptografi. Informatika. Bandung, Indonesia (2006)

[11] Reque, J. J., Mingeut, J. M.: SLSB : Improving the Steganographic Algorithm LSB. International Journal of Engineering Science and Computing. pp. 8137 (2009)

[13] M. Mu, L. B.: Multimedia Data Hiding. Springer-Verlag. New York, USA (2003)

[14] Fridrich J, G. M.: Practical Steganalysis of Digital Images-State of the Art. Dartmount (2006)

[15] Juan Jose, J. M.: SLSB : Improving the Steganographic Algorithm LSB (2009)

[16] Sugiyono, P. D.: Metode Penelitian Kuantitaf, Kuantitatif, dan R\&D. Alfabeta Bandung, Indonesia (2012) 